\title{
Kommunikationsanalytische Ansätze zur Beschreibung diskursiver Lagerbildung in der Corona-Krise
}

\author{
Linguistic Communication Analysis of Discursive Antagonisms \\ in the Corona Crisis
}

\begin{abstract}
ZUSAMMENFASSUNG
Die Corona-Krise hat zu schweren inneren Konflikten und gesellschaftlicher Spaltung in vielen Ländern der Welt geführt. Diese Spaltung wird zu einem großen Teil durch antagonistische diskursive Praktiken hergestellt, vertieft und reproduziert. Dieser Beitrag unternimmt den Versuch die theoretischen Grundlagen für die Analyse und Beschreibung der konkurrierenden Diskurslager und die Bedingungen ihrer Entstehung zu erarbeiten. Dabei sollen die theoretische Trennlinie zwischen der traditionellen politischen Diskurstheorie under der linguistischen Kommunikationsanalyse aufgehoben und vielversprechende methodische Ansätze für die Analyse multimodaler Ressourcen und ihres kommunikativen Gebrauchs in den mit der Corona-Krise assoziierten Diskursen aufgezeigt werden.

Schlüsselwörter: Diskurstheorie, Kommunikationsanalyse, Linguistik, Corona, Narrativ
\end{abstract}

\section{ABSTRACT}

The Corona crisis has led to internal conflict and the creation of enormous societal chasms in many countries of the world that would have been unthinkable a year ago. This divide is in large part being created, reproduced and deepened through ongoing antagonistic discursive practices. This article aims at outlining the theoretical groundwork for the analysis and description of the resulting discursive camps and the facilitating processes. It endeavors to bridge the theoretical gap between traditional political discourse theory and linguistic communication analysis and show promising methodological pathways for forensic analysis of multimodal resources and their communicative use in the current Corona associated discourses.

Keywords: discourse theory, communication analysis, linguistics, corona crisis, narrative

\section{Epidemie der gesellschaftlichen Spaltung}

Die öffentliche Kommunikation und der mediale Alltag nahezu aller Länder der Welt wurde im Jahr 2020 thematisch von einem Ereignis bestimmt, dessen Bewertung und Einordnung zu massiven Veränderungen der politischen Ordnung sowie

Dennis Kaltwasser, Institut für Germanistik, Justus-Liebig-Universität Gießen, Otto-Behaghel-Str. 10B, 35394 Gießen, dennis.kaltwasser@germanistik.uni-giessen.de, https://orcid.org/0000-0002-4097-637X 
zu einer gesellschaftlichen Spaltung ungekannten Ausmaßes in den Bevölkerungen dieser Länder geführt hat: das Auftreten des Virus SARS-COV-2 und der davon ausgelösten Atemwegserkrankung COVID-19. Als Auslöser der diskursiven Polarisierung spielt dabei zumindest im deutschen Sprachraum das medizinische Phänomen selbst eine eher untergeordnete Rolle. Der Streit entfacht sich vielmehr an der Informationspolitik staatlicher Institutionen, der massenmedialen Aufbereitung der Krise und vor allem an der Beurteilung der weitreichenden politischen Maßnahmen, die zu seiner Bewältigung eingeleitet wurden.

Auffällig ist dabei zunächst, dass es weder im politischen Feld, insbesondere in den Parlamenten, noch in den Leitmedien zu einer abwägenden oder gar kontroversen Diskussion dieser Maßnahmen gekommen ist. Eine rationale Aushandlung verschiedener Sichtweisen auf das Ereignis, die zu den konstitutiven Konfliktlösungsprozessen einer deliberativen Demokratie gehören würde (vgl. Benhabib, 2000; Cohen, 1997), konnte bislang kaum stattfinden. Stattdessen müssen wir auch im elften Monat der Krise noch immer eine Polarisierung des Diskurses mit zwei einander unversöhnlich gegenüberstehenden Lagern feststellen. Auch die Wissenschaftskommunikation ist von diesem Phänomen erfasst, was beispielsweise den Tübinger Geologen Thomas Aigner zum Austritt aus der Mainzer Akademie der Wissenschaften ${ }^{1}$ bewog, den er mit den mahnenden Worten begründet:

\begin{abstract}
Wo bleibt ein früher üblicher breiter Diskurs mit einer ausgewogenen Würdigung der teilweise sehr konträren Wortmeldungen von Wissenschaftlern und Ärzten verschiedener Fachrichtungen, Juristen, Psychologen, Soziologen, Ökonomen und Philosophen? Warum gibt es keine Reaktion der Akademien, wenn in den letzten Monaten immer wieder Stimmen von ausgewiesenen Fachleuten (oftmals von internationalem Rang), die eine vom Einheits-Narrativ abweichende, ja teilweise diametral widersprechende Einschätzung artikulieren, ignoriert, ausgegrenzt, ja sogar diffamiert, zensiert und in sozialen Medien gelöscht werden? [...] Ich kann es mit meinem Gewissen nicht vereinbaren, ein Teil dieser Art von Wissenschaft zu sein. Ich möchte einer Wissenschaft dienen, die einer faktenbasierten Aufrichtigkeit, einer ausgewogenen Transparenz, und einer umfassenden Menschlichkeit verpflichtet ist (AI 01).
\end{abstract}

Bereits im April des Jahres konstatiert die Nachrichtenredaktion von RTL unter der Dachzeile „Deutschland teilt sich in zwei Lager“: „Es geht ein Riss durch Deutschland, durch Freundeskreise, durch Nachbarschaften, manchmal sogar durch Familien“ (RT 01). Die Beschreibung dieser zwei großen gesellschaftlichen Lager und die Rekonstruktion ihrer konstitutiven Merkmale aus der diskursiven Praxis gehören zu den Aufgaben des vorliegenden Beitrags, man könnte jedoch in einem ersten Zugriff vereinfachend von „Maßnahmenbefürwortern“ auf der einen und „Maßnahmenkritikern“ auf der anderen Seite sprechen. Wie tief diese Spal-

1 Auch ein Mitglied der nationalen Akademie der Wissenschaften Leopoldina, Michael Esfeld, warnt in diesem Zusammenhang vor Szientismus und dem politischen Missbrauch der Wissenschaft, die er aktuell in der Rolle „der Staatsreligion in vor-aufklärerischer Zeit“ sieht (ND 02). 
tung auch in den politischen und journalistischen Alltag eingedrungen ist, wird exemplarisch deutlich, wenn einerseits etwa die Bundesvorsitzende einer Regierungspartei, Saskia Esken, hunderttausende Teilnehmer einer Berliner Demonstration als „Covidioten“ (TW 01) bezeichnet, Margitta Heinecke, Richterin am Verwaltungsgericht Berlin und Bundesdelegierte einer Oppositionspartei, dazu auffordert, Kritiker der Maßnahmen ,gesellschaftlich zu ächten“ und vom Arbeitsmarkt auszuschließen (TW 02), Bundeskanzlerin Merkel Kritiker der Maßnahmen pathologisiert und in diesem Zusammenhang gar von einem „Angriff auf unsere ganze Lebensweise" spricht (SP 01) und andererseits Anselm Lenz, ehemaliger Redakteur der Tageszeitung taz, von einem „Corona-Faschismus“ (KF 01) und Walter van Rossum, Redakteur des WDR, vom „Tod der Aufklärung“" (ND 01) spricht.

Es handelt sich bei den Ereignissen seit März 2020 insgesamt um ein vielschichtiges Phänomen mit unabsehbar weitreichenden gesellschaftlichen Folgen. Als solches wird es zukünftig sicher ein zentraler Gegenstand aller Bereiche der Geistes- und Sozialwissenschaften werden und seine angemessene Untersuchung kann zu großen Teilen nur interdisziplinär erfolgen. Der Fokus des vorliegenden Beitrags liegt daher auf der Frage, welchen Beitrag die Linguistik und insbesondere die linguistische Kommunikationsanalyse zur Beschreibung gegenwärtiger diskursiver Phänomene, die zur Bildung antagonistischer Diskursgemeinschaften führen, leisten kann. Hierfür sollen aus diskurs- und handlungstheoretischer Perspektive verschiedene methodische Zugänge zur Beschreibung lokaler und globaler Diskursgemeinschaften erschlossen und zueinander in Beziehung gesetzt werden. Konkrete, hermeneutisch abgestützte Analysen würden den Rahmen des vorliegenden Beitrags sprengen, insofern kann hier nur der theoretisch-methodische Rahmen entworfen und zur weiteren Forschungsarbeit angeregt werden.

\section{Text- und diskurstheoretische Grundlagen}

Ausgangspunkt der vorliegenden Untersuchung ist die Annahme, dass man Texte als Werkzeuge des sprachlichen Handelns auffassen kann (vgl. Gloning, 2010). Daraus folgt, dass die textlinguistisch relevanten Beschreibungsaspekte von Texten bzw. multimodalen Kommunikationsmitteln nicht losgelöst von ihrem Verwendungszusammenhang betrachten werden. Im Zentrum der handlungstheoretischen Analyse stehen nicht nur Äußerungen wie Texte, Gesprächsbeiträge usw. selbst, sondern vielmehr die kommunikativen Zusammenhänge, in denen sie von konkret Handelnden mit bestimmten Absichten unter spezifischen Bedingungen für konkrete Zwecke verwendet und dann in bestimmter Weise verstanden (oder auch missverstanden) werden und die ihrerseits mit Formen der sozialen Praxis verflochten sind (vgl. Fritz, 1982, S. 13-23; Luckmann, 1986, S. 199-206; Strecker, 1987, S. 28-64). Damit werden nicht nur zentrale Analyseeinheiten wie Textfunktion oder Thema zu Größen des Meinens und Verstehens. Gleichzeitig 
rücken auch vielfältige Wissensaspekte als Grundlage der Erzeugung kommunikativen Sinns in den Vordergrund und die Beschreibungsmöglichkeiten erstrecken sich auch auf komplexe, multimodale, institutionell gebundene und medial vermittelte Kommunikation (vgl. Fritz, 2016, S. 7), wodurch ein niederschwelliger Anschluss an diskurstheoretische Zugänge auch aus den Feldern der Politik- und Sozialwissenschaft möglich wird. In einem solchen theoretischen Bezugsrahmen können zwei analytische Ansätze gewinnbringend aufeinander bezogen werden: Auf der globalen Ebene können Diskurskoalitionen auf der Grundlage des gemeinsamen Bezugs zu bestimmten Diskursen beschrieben werden, während auf der lokalen Ebene die konkrete sprachliche Reproduktion verschiedener Aspekte dieser Diskurskoalitionen gezeigt werden kann und das kommunikative Handeln der Diskursteilnehmer auf dieser Folie verständlich wird.

Zur Bearbeitung der globalen Ebene muss zunächst der in der Literatur zuweilen recht heterogen gebrauchte Diskursbegriff abgegrenzt werden. Ich orientiere mich zu diesem Zweck in einem ersten Schritt an den weitgehend miteinander verträglichen Diskursdefinitionen von Dryzek (2013) und Hajer (1993) und beziehe diese anschließend auf die Kategorien und Beschreibungsverfahren der linguistischen Kommunikationsanalyse.

A discourse is a shared way of apprehending the world. Embedded in language, it enables those who subscribe to it to interpret bits of information and put them together into coherent stories or accounts. Discourses construct meaning and relationships, helping define common sense and legitimate knowledge. Each discourse rests on assumptions, judgements, and contentions that provide the basic terms for analysis, debate, agreements, and disagreements (Dryzek, 2013, S. 9).

Discourse is $[\ldots]$ defined as an ensemble of ideas, concepts and categories through which meaning is given to phenomena. Discourses frame certain problems; that is to say, they distinguish some aspects of a situation rather than others. [...] Discourse [...] forms the context in which phenomena are understood and thus predetermines the definition of the problem (Hajer, 1993, S. 45).

Beiden Definitionen ist gemein, dass sie Diskurse als eine sozial verankerte komplexe Ordnung auffassen, welche die Wahrnehmung von Phänomenen bzw. Weltausschnitten strukturieren. Sie bilden damit die Grundlage für die soziale Konstruktion von Problemen, ihre kollektive Analyse und auf sie bezogene Debatten. Sie regulieren zudem, wie einzelne Ereignisse und Sachverhalte zusammenhängen, welche inhaltlichen Positionen als legitimes Wissen gelten können und mit dem ,gesunden Menschenverstand" vereinbar sind.

Im Hinblick auf ihr Gegebensein versteht Hajer (1993) Diskurse als „,ideas, concepts and categories" (S. 45) und verortet sie damit auf der epistemischen Ebene. Allerdings können diese nur auf der Äußerungsebene im weitesten Sinn sichtbar und damit auch reproduzierbar und analysierbar werden. Darauf weist auch Dryzek (2003) hin, wenn er den Diskurs als ,embedded in language“ (S. 9) charakterisiert. Dies stellt jedoch eine unnötige Verengung dar, denn neben Spra- 
che können vielfältige multimodale Ressourcen (Bild, Film, Ton, Ikonographie, Kleidung, Gesten usw.) zur Erzeugung kommunikativen Sinns genutzt werden. Auch der durch soziale oder institutionelle Praktiken erzeugte Sinn kann Bestandteil eines Diskurses sein, ebenso jener, der durch Gesetze, die Anwendung bestimmter Techniken oder auch durch architektonische Elemente im öffentlichen Raum entsteht. So ist etwa das kollektive Tragen einer Maske im Alltag (unter anderem) verstehbar als Hinweis auf eine allgegenwärtige Ansteckungsgefahr ${ }^{2}$.

Aufbauend auf dieser skizzenhaften Definition lassen sich die oben umrissenen antagonistischen Lager als Diskurskoalitionen auffassen. Diese beschreibt Hajer (1993, S. 47) als ,a group of actors who share a social construct [which consists of] a set of story lines, the actors that utter these story lines and the practices that conform to these story lines, all organized around a discourse“. Dabei spielt das oben erwähnte partielle Verstehen oder auch das Missverstehen häufig eine wichtige Rolle für die Koalitionenbildung, wie Hajer (2006, S. 69) betont:

[The] assumption of [universal] mutual understanding is false. Discourse analysis brings out, time and again, that people talk at cross-purposes, the people do not really or do not fully understand each other. This is a fact of life but, interestingly, this can be very functional for creating a political coalition.

Die vorangehenden Überlegungen eröffnen die Möglichkeit zur systematischen Rekonstruktion solcher Diskurskoalitionen auf der Grundlage einer kommunikationsanalytischen Untersuchung relevanter Corpora im Hinblick auf den Gebrauch kommunikativer Ressourcen, die Hinweise auf den ,Common Ground“ einer Diskurskoalition geben. Hierzu gehören reproduzierte Narrative, Aspekte des geteilten Wissens, vor allem über in der Diskursgemeinschaft etablierte Fakten und ihren Zusammenhang, sowie konstitutive Merkmale von Gruppenidentitäten. Diese können definiert werden über geteilte Normen, Werte und deren legitime Repräsentionsformen sowie über Annahmen gesellschaftlicher Ziele und Prioritäten.

\section{Aspekte der kommunikationsanalytischen Rekonstruktion und Beschreibung von Diskurskoalitionen}

Nachdem die Bedeutung von Diskursen für die Abgrenzung der in der Einleitung vorläufig als „Maßnahmenbefürworter“ und Maßnahmenkritiker“ bezeichneten gesellschaftlichen Lager deutlich geworden ist, können wir diese als spezifische Diskurskoalitionen näher definieren. Als Arbeitshypothese erscheint es aussichtsreich, für das erste der beiden Lager einen „Gesundheitsgefahrendiskurs“ und

2 Ob sich der Träger einer Maske in einer konkreten Interaktion auf diese Annahme festlegen will, ist für die Erzeugung des kommunikativen Sinns irrelevant, da sich bereits der Verordnungsgeber darauf festgelegt hat. Anders scheint es sich daher bei individuellen thematisch interpretierbaren Praktiken wie etwa Hamsterkäufen zu verhalten, die eher Symptomcharakter haben. 
einen „Solidaritätsdiskurs“ als bestimmend anzunehmen, während man für das zweite Lager versuchen könnte, einen „Demokratiegefährdungsdiskurs“ und einen „Unverhältnismäßigkeitsdiskurs“ nachzuzeichnen. Im Folgenden sollen zunächst einige Hinweise zur Zusammenstellung eines geeigneten Corpus gegeben werden und anschließend kommunikationsanalytische Verfahren der Rekonstruktion diskursiver Praktiken, die für das erste der beiden Lager konstitutiv sind, illustriert werden.

\subsection{Onlineforen und journalistische Texte als empirische Datenbasis für die kommunikationsanalytische Rekonstruktion von Diskurskoalitionen}

Für die Rekonstruktion und Dokumentation diskursiver Phänomene stellt die Kommunikation in Onlineforen im Kontext journalistischer und anderer Onlineangebote aus mehreren Gründen eine sehr ertragreiche und methodisch attraktive Datenbasis dar. Zunächst ist sie geprägt von den Affordanzen (vgl. Hutchby, 2001), die sich aus dem jeweils spezifischen Design und der technischen Ausgestaltung der Autorenumgebung innerhalb der Webanwendung ergeben. Das Onlineforum als Medienformat kann man dabei als einen technisch-medialen Möglichkeitsraum (vgl. Bucher, Gloning, \& Lehnen, 2010) sehen, der seine kommunikative Nutzung zwar nicht determiniert, jedoch je nach seiner konkreten Realisationsform bestimmte Nutzungsmöglichkeiten eröffnet und andere verschließt. Das Resultat der kommunikativen Nutzung einer solchen Umgebung ist eine Form des schriftlichen Gruppengesprächs, das Marcoccia (2004, S. 116) als „written conversation“ charakterisiert. Im Kontrast zu dyadischer Kommunikation handelt es sich dabei um Polyloge mit komplexen Bezugshierarchien und Rollenkonstellationen (zum Polylogbegriff vgl. Kerbrat-Orecchioni 2004). Von besonderem Interesse für die resultierenden Interaktionsstrukturen sind hierbei die Möglichkeiten des Zitierens und der Bezugnahme auf vorangegangene Forenbeiträge sowie die Visualisierung der resultierenden Beitragsstruktur (vgl. Kaltwasser, 2019, S. 168-173).

Das Forum des Onlinemagazins Telepolis bietet eine übersichtliche und benutzerfreundliche hierarchische Baumdarstellung zur Navigation von Diskussionsthreads und unterstützt dadurch auch komplexere Interaktionsstrukturen. Andererseits äußert sich hier ein heterogenes Publikum über den gesamten Untersuchungszeitraum hinweg sehr detailliert und mit einem breiten perspektivischen Spektrum zu verschiedenen Teilthemen der relevanten Diskurse im thematischen Zusammenhang mit der Corona-Krise, weshalb dieses Forum auch als ein zentraler Bestandteil des Textbasis ausgewählt wurde. Neben journalistischen Texten und der Forenkommunikation in ihrem Umfeld kann sich jedoch auch die Analyse von Texten aus anderen Domänen, etwa der internen Kommunikation politischer Institutionen, als ertragreich erweisen, wie der nächste Abschnitt zeigt. 


\subsection{Narrative und story lines}

Eine wichtige Einsicht der Erzählforschung besteht in der Erkenntnis, dass Geschichten immer zu einem bestimmten Zweck erzählt werden und dieser Zweck einen strukturierenden Einfluss darauf hat, wie und mit welchen Mitteln erzählt wird (vgl. Fritz, 1982, S. 295-307). Auf diesen Zusammenhang zwischen Erzählstrategie und weiterführenden Intentionen verweisen auch Jacobs und Sobieraj (2007, S. 6):

For any given narrative, it is possible to investigate the pragmatic goals the narrator is pursuing, the institutional context in which the story is being told, and the narrative conventions that regulate the talk or writing taking place within that specific institutional context.

Worin diese „pragmatic goals“ in der politischen Kommunikation bestehen können, konkretisieren Homolar und Rodríguez-Merino (2019, S. 562) zusammenfassend:

$[\mathrm{N}]$ arratives have a deeper function in society and politics than simply being one of many communicative ways for "someone telling someone else that something happened" [...] "strategic narratives" provide a tool for political agents to shape behavior [...] and the "discursive environment in which they operate" [Hervorhebung original].

Für den Gesundheitsgefahrendiskurs ist die Regierung einer der wichtigsten Akteure der öffentlichen Kommunikation und somit wesentlich an der Etablierung dominanter Narrative beteiligt. Dass auch sie in Übereinstimmung mit ihren weitergehenden Intentionen reflektiert eine spezifische Erzählstrategie wählt, geht aus einem zunächst geheimen Strategiepapier des Bundesinnenministeriums ${ }^{3}$ hervor, das als übergeordnetes Ziel der Kommunikationsstrategie festlegt, „die Maßnahmenplanung der Bevölkerung [zu] vermitteln“. Zu diesem Zweck werden zunächst abstrakte Kernelemente der Erzählung formuliert:

Der Worst Case ist mit allen Folgen für die Bevölkerung in Deutschland unmissverständlich, entschlossen und transparent zu verdeutlichen. [...] Die Vermeidung des Worst Case ist als zentrales politisches und gesellschaftliches Ziel zu definieren (AW 01).

Diese werden anschließend in konkrete Richtlinien übersetzt, die auch die gezielte Ausblendung bestimmter Informationen beinhaltet, die zu einem unerwünschten Verständnis der Erzählung führen könnte:

Wir müssen wegkommen von einer Kommunikation, die auf die Fallsterblichkeitsrate zentriert ist. [...] Diese Mechanismen haben in der Vergangenheit sicher zur Verharmlosung der Epidemie beigetragen (AW 01).

3 Das Bundesinnenministerium hat eine Fassung des zunächst als Verschlusssache eingestuften Papiers am 28. April 2020 auf seiner Internetseite zugänglich machen müssen (BI 01). Im Zuge einer Auskunftsklage gegen die Bundesrepublik Deutschland konnte zudem der zugrundeliegende E-Mail-Verkehr zur Entstehung des Papiers öffentlich gemacht werden (SM 01). 
Nachdem der Zweck der Erzählung definiert und zentrale strategische Elemente diskutiert wurden, erhalten die Akteure der öffentlichen Kommunikation auch konkrete Handlungsanweisungen, Erzählbausteine und Hinweise auf gewünschte Zielgruppen für das zukünftige Narrativmanagement:

Um die gewünschte Schockwirkung zu erzielen, müssen die konkreten Auswirkungen einer Durchseuchung auf die menschliche Gesellschaft verdeutlicht werden: Viele Schwerkranke werden von ihren Angehörigen ins Krankenhaus gebracht, aber abgewiesen, und sterben qualvoll um Luft ringend zu Hause. Das Ersticken oder nicht genug Luft kriegen ist für jeden Menschen eine Urangst. Die Situation, in der man nichts tun kann, um in Lebensgefahr schwebenden Angehörigen zu helfen, ebenfalls. [...] Wenn [Kinder] ihre Eltern anstecken, und einer davon qualvoll zu Hause stirbt und sie das Gefühl haben, Schuld daran zu sein, weil sie z.B. vergessen haben, sich nach dem Spielen die Hände zu waschen, ist es das Schrecklichste, was ein Kind je erleben kann (AW 01).

Wichtige Bestandteile der Erzählung behandeln also nicht nur das, was bisher geschehen ist, sondern vor allem, wie das bisher Geschehene mit dem zusammenhängt, was noch geschehen könnte. Ein zweiter zentraler Topos des Gesundheitsgefahrendiskurses, der hier in der Andeutung der Abweisung im Krankenhaus anklingt, ist die drohende Überlastung der Intensivstationen ${ }^{4}$, die sowohl in der medialen Berichterstattung als auch in Argumentationen über die Angemessenheit der Maßnahmen häufig eine wichtige Rolle spielt.

Es ist unmittelbar einsichtig, dass bereits die Frage der Akzeptanz oder Ablehnung dieses Narrativs, das auf die Aktivierung von „Urängsten“ und Schuldgefühlen von Kindern abzielt, zu einer fundamentalen Spaltung der Gesellschaft führen muss. Auch die hier angestrebte Ausblendung der Fallsterblichkeitsrate, die je nach Informationsstand zu unverträglichen Sichtweisen auf das epidemische Geschehen führt, trägt bereits den Keim für die Entstehung antagonistischer Lager in sich. Dies umso mehr, da gerade die Kenngröße der Fallsterblichkeit in engem Zusammenhang mit der Plausibilität der drohenden Zukunftsszenarien steht und in der Forenkommunikation zu den am schwersten umkämpften Wissensbeständen gehört.

Dies wirft sowohl strategische als auch kommunikationsethische Fragen auf, die auch für den Gesundheitsgefahrendiskurs und die resultierende verhärtete Lagerbildung von zentraler Bedeutung sind. Dahlstrom (2014, 13617) bemerkt: „Little is known about the expectations that audiences hold with regard to how science should be communicated to them. [...] How does science communicated in narrative format influence audience perceptions of trust? [...] Do narratives decrease trust because they are seen as overly sensational or manipulative?" Er sieht

4 Diese wurde von Bundeskanzlerin Merkel bereits am 11. März in einer Ansprache in Aussicht gestellt, in der es heißt, das Vorgehen in Deutschland sei davon bestimmt, dass unser Gesundheitssystem nicht überlastet werde (BR 01). 
zudem die intellektuelle Autonomie des Publikums als Grundprinzip deliberativer Prozesse gefährdet:

Narratives are intrinsically persuasive. Because they describe a particular experience rather than general truths, narratives have no need to justify the accuracy of their claims; the story itself demonstrates the claim. [...Results from t] he field of narrative persuasion generally suggest that audiences are more willing to accept normative evaluations from narratives than from more logical-scientific arguments. [...] In fact, accepted narratives are trusted so much that individuals rarely allow evidence to contradict the narrative; evidence is altered to fit their narratives (Dahlstrom, 2014, 13616)

Diese von beiden Diskurslagern im jeweils gegnerischen Lager wahrgenommene Faktenresistenz bzw. einseitige „Faktendiät" wird in den Foren regelmäßig kritisiert und hat zu der häufig gebrauchten abwertenden Kennzeichnung Zeugen Coronas geführt, wie Beleg (1) zeigt ${ }^{5}$ :

(1)

Chekow

Am 22. September 2020 um 09:26

Und Sie als „Zeuge Coronas“ glauben ausschließlich, was Ihnen „Herr Drosten“ sagt? So funktioniert Meinungsbildung weder in der Wissenschaft noch im Alltag. [...] (TS 01)

Beleg (2) aus einem Forenverlauf zu einem Telepolis-Magazinbeitrag des Psychologen Christof Kuhbandner von der Universität Regensburg mit dem Titel „Von fragwürdigen und fehlinterpretierten Corona-Zahlen“ kann, stellvertretend für zahllose weitere Kommentare, den engen Zusammenhang zwischen den oben diskutierten Teilaspekten und der so erzeugten diskursiven Trennung illustrieren:

(2)

Don Obi 24.12.2020 18:00

\section{Kuhbandners Querdenker-Unfug}

War ja klar. Baghdi-Jünger und Realitätsleugner Kuhbandner verbreitet mal wieder mit wilden Zahlenspielereien absoluten Unfug. [...]

$>$ Anders als von vielen Medien, in Regierungserklärungen und von

$>$ manchen Wissenschaftlern der Anschein erweckt wird, gibt es

$>$ demnach keinen Grund, außergewöhnliche Ängste zu haben und

$>$ bisher nie dagewesene Maßnahmen zu ergreifen

Es gibt eine Vielzahl von Gründen für „außergewöhnliche Ängste“. Eine Corona-Erkrankung ist in Symptomatik und Therapie auch bei nicht-tödlichem Verlauf der kaum vorstellbare Horror, welcher auch nach mehreren Wochen oder Monaten nicht zu Ende ist [Hervorhebung original] (TP 02).

\footnotetext{
5 Alle Hervorhebungen in Belegtexten sind original.
} 
Deutlich erkennbar wird hier eine Lagerzuweisung vorgenommen („Querdenker"), es wird dem Gegenüber die Rationalität abgesprochen („Unfug“, „,Realitätsleugner"), es wird eine Anfälligkeit für Autoritätstrugschlüsse unterstellt („Baghdi[sic.]-Jünger") und ein emotionaler Ausschnitt des bereits diskutierten Narrativs und angegliederter story lines wird faktizierend reproduziert (,,er kaum vorstellbare Horror“, „nach mehreren Wochen oder Monaten nicht zu Ende“).

\subsection{Kollektividentitäten}

Neben der Untersuchung dominanter Narrative bietet die Rekonstruktion von Kollektividentitäten aufgrund beobachtbarer diskursiver Praxis vielfache Möglichkeiten zur kommunikationsanalytisch fundierten Analyse der Diskursgemeinschaften. Einen ersten Zugang bieten hier die (teils recht kreativen) Formen der Bezugnahme auf das jeweils eigene und vor allem das gegnerische Lager. Eine kurze, bei weitem nicht vollständige Liste kann einen ersten Eindruck vermitteln: Covidiot, Corona-Leugner, Schwurbler, Aluhutträger, Verschwörungstheoretiker, Querdenker, Bhakdi-Jünger, Impfgegner, Antisemit, Nazi, Reichsbürger, Esoteriker. Und auf der anderen Seite: Zeuge Coronas, Coronazi, Maskennazi, Faschist.

Besonders aufschlussreich ist auch die Identifikation mit gruppenspezifischen Werten und den legitimen Formen ihres Ausdrucks. Für das Lager der Maßnahmenbefürworter spielt der Wert der Solidarität eine zentrale Rolle. Hierfür finden sich zahlreiche Belege, u.a. bereits in dem oben diskutierten Strategiepapier des Innenministeriums. Am eindrücklichsten wird die Idee der Maßnahmenbefürwortung als legitimer Ausdruck von Solidarität vielleicht bei einer Demonstration sichtbar, auf der Mitglieder der Jugendorganisation einer Oppositionspartei ein Banner mit der Aufschrift „Impfen ist Liebe“ zeigen (RD 01). Ein zentraler Topos der argumentativen Auseinandersetzung zwischen den Lagern ist dabei weniger die Frage, ob Solidarität ein geteilter Wert ist, sondern eher, was als legitimer Ausdruck von Solidarität gelten kann. Die Belege (3) bis (5) geben hier einen Einblick:

(3) -su-, 3.1.2021 15:35

Re: Bitte alle im Forum Patientenverfügung unterschreiben

SKR700 schrieb am 3.1.2021 14:36:

$>$ Die Corona-Leugner, die hier so laut herumtönen sollen bitte eine

$>$ Patientenverfügung unterschreiben, wonach sie auf jegliche

$>$ medizinische Versorgung verzichten und anderen den Vortritt

$>$ lassen. Gibt ja sowieso keine Viren. [...]

Schon blöd, wenn die Fakten eine andere Sprache sprechen, als Sie es sich wünschen. Deshalb wollen Sie jeden, der diese Fakten nennt, bestrafen. Ganz schön komisches Weltbild, das das Team „Solidarität"“ da hat. „Solidarität" nur mit solchen Menschen, die zu allen Ja und Amen 
sagen. Das jeden kritischen Geist bestrafen will bis hin zum Verweigern medizinischer Hilfe (TP 03).

(4)

WhenSeptemberEnds, 18.12.2020 18:29

Wer sich nicht impfen lässt, ist ein rücksichtsloser Egoist.

Unfassbar, wie niedrig das Verantwortungsbewußtsein der Covidioten ist. Sie setzen ihr Umfeld bewußt einem tödlichen Risiko aus. Das Gemeinschaftsgefühl und die Empathie ist in asiatischen Ländern weitaus ausgeprägter. Bei so vielen Idioten in diesem Land würde ich gern zu meinen Schwiegereltern in Japan fliegen (TP 04).

(5)

aqudraht, 16.12.2020 11:34

Ein arroganter Kleinbürger faselt von Solidarität

[...] Das Geschwurbel von Solidarität aber ist das Obszönste. [...] Auch im Rahmen der Pandemie wurde Hartz-Beziehern jede Entlastung verweigert, wurden notwendige Einbauten in Schulen versäumt, der Abbau im Pflege- und Krankenhausbereich in der Pandemie weitergeführt. Hunderte von Milliarden ,für die Wirtschaft“ herausgeschmissen. Da hätte es Solidarität gebraucht, da wäre sie noch zu fordern. [...] „Solidarität“ beim zwangsweisen Einsatz weitgehend ungetesteter Medikamente einzufordern ist schlicht unverschämt. Niemand kann sagen, ob die Impfung Infektionen verhindert, wie lange mögliche Immunitäten anhalten, welche langfristigen Folgen zu erwarten sind, wie die Präparate in Kombination mit anderen Medikamenten, bei Schwangeren oder Multimorbiden wirken. Da sind sie nämlich nicht getestet worden (TP 04).

\subsection{Weitere kommunikationsanalytische Zugänge}

In Ergänzung zur Rekonstruktion von geteilten Narrativen und der Beschreibung von Gruppenidentitäten gibt es noch eine Reihe weiterer Ansatzpunkte zur Rückbindung der diskursiven Lager an das konkrete sprachliche Handeln ihrer Mitglieder. Zu ihnen gehört die Beschreibung von Kollektivakteuren, die etwa in Onlineforen gemeinsam die Rolle eines einzelnen Akteurs in bestimmten Kommunikationsformen übernehmen (für die Vorwurfskommunikation und das Gemeinsam Planen vgl. Kaltwasser, 2019, S. 114-160; für die Rolle des ,kollektiven Antagonisten" in der Argumentation vgl. Lewiński, 2011). Gerade in argumentativen Interaktionen lassen sich außerdem eine ganze Reihe aufschlussreicher Elemente des ,Common Ground“ einer Diskursgemeinschaft hermeneutisch herausarbeiten. Dies betrifft insbesondere geteilte Wissensbestände und Wertvorstellungen, die entweder in stützenden Zügen explizit gemacht werden oder die als vorausgesetzte Schlusspräsuppositionen oder Bewertungsprinzipien nachvollzogen werden können sowie gängige Argumentationsmuster und Beweisprozeduren. Der häufig anzutreffende Rückgriff auf das argumentum ad verecundiam sowie die Kritik daran geben beispielsweise einen guten Überblick über die wichtigsten Exponenten und Gewährsleute der jeweiligen Lager. 


\section{Fazit}

Das Ziel des vorliegenden Beitrags bestand darin, Ansätze zur handlungstheoretisch fundierten, detaillierten und textgestützten Untersuchung jener Diskurse vorzustellen, die thematisch eng mit der Corona-Krise verbunden sind und die eine konstitutive Rolle für die Erzeugung der aktuell vorhandenen antagonistischen Diskursgemeinschaften spielen. Der zur Verfügung stehende Raum lässt dabei keine detaillierten Analysen zu, insofern konnten nur Anschlussstellen identifiziert und „Absprungmarken“ für zukünftige Untersuchungen herausgearbeitet werden. Es sind dies: a) der erzähltheoretische Zugang zur Untersuchung dominanter Narrative, b) die sprachgestützte Analyse und Beschreibung von Kollektividentitäten, c) die Beschreibung der sprachlichen Aktivitäten von Kollektivakteuren in unterschiedlichen Kommunikationsformen, insbesondere der Argumentation und d) die Rekonstruktion von geteilten Wissensbeständen und Beweisverfahren. Ergänzend wurden Onlineforen als methodisch attraktive Datenbasis für diese Untersuchungen vorgestellt.

\section{References}

Benhabib, S. (2000). The Embattled Public Sphere. Hannah Arendt, Jürgen Habermas and Beyond. In E. Ullmann-Margalit (Ed.), Reasoning Practically (pp. 164-181). Oxford: Oxford University Press.

Bucher, H. J., Gloning, T., \& Lehnen, K. (2010) Medienformate: Ausdifferenzierung und Konvergenz - Zum Zusammenhang von Medienwandel und Formatwandel. In H. J. Bucher, T. Gloning, K. Lehnen (Eds.), Neue Medien - Neue Formate. Ausdifferenzierung und Konvergenz in der Medienkommunikation (pp. 9-40). Frankfurt am Main: Campus Verlag.

Cohen, J. (1997). Deliberation and Democratic Legitimacy. In J. Bohman, \& W. Rehg (Ed.), Deliberative Democracy. Essays on Reason And Politics (67-91). Cambridge, MA: MIT Press.

Dahlstrom, M. (2014). Using narratives and storytelling to communicate science with nonexpert audiences. PNAS, 16(4), 13614-13620. DOI: 10.1073/pnas.1320645111.

Dryzek, J. (2013). The Politics of the Earth. Environmental Discourses. Oxford: Oxford University Press.

Fritz, G. (1982). Kohärenz. Grundfragen der linguistischen Kommunikationsanalyse. Tübingen: Narr.

Fritz, G. (2016). Zur linguistischen Analyse von Diskursen. Eine handlungstheoretische Perspektive. In G. Fritz (Ed.), Beiträge zur Texttheorie und Diskursanalyse (pp. 7-44). Gießen: Giessener Elektronische Bibliothek.

Gloning, T. (2010). Funktionale Textbausteine in der historischen Textlinguistik. Eine Schnittstelle zwischen der Handlungsstruktur und der syntaktischen Organisation von Texten. In A. Ziegler, \& Ch. Braun (Eds.), Historische Textgrammatik und historische Syntax des Deutschen: Traditionen, Innovationen, Perspektiven (pp. 173-194). Berlin: De Gruyter.

Hajer, M. (1993). Discourse coalitions and the institutionalisation of Practice. The case of acid rain in britain. In J. Forester, \& F. Fischer (Eds.), The Argumentative Turn in Policy and Planning (pp. 43-76). London: UCL Press.

Hajer, M. (2006). Doing discourse analysis: coalitions, practices, meaning. In M. van den Brink, \& T. Metze (Eds.), Words matter in policy and planning. Discourse theory and method in the social sciences (pp. 65-74). Utrecht: KNAG/NETHUR.

Homolar, A., \& Rodríguez-Merino, P. A. (2019). Making sense of terrorism: a narrative approach to the study of violent events. Critical Studies on Terrorism, 12(4), 561-581. 
Hutchby, I. (2001). Technologies, Texts and Affordances. Sociology, 35(2), 441-456.

Jacobs, R. N., \& Sobieraj, S. (2007). Narrative and Legitimacy: U.S. Congressional Debates about the Nonprofit Sector. Sociological Theory, 25(1), 1-25.

Kaltwasser, D. (2019). Forenkommunikation in Onlinezeitungen. Pressekommunikation im medialen Wandel. Gießen: Gießen University Library Publications.

Kerbrat-Orecchioni, C. (2004). Introducing Polylogue. Journal of Pragmatics, 36, 1-24.

Lewiński, M. (2011). The Collective Antagonist. Multiple Criticism in Informal Online Deliberation. In F. M. van Eemeren, B. Garssen, D. Godden, \& G. Mitchell (Eds.), The Seventh Conference of the International Society for the Study of Argumentation (pp. 1089-1101). Amsterdam: SicSat.

Luckmann, T. (1986). Grundformen der gesellschaftlichen Vermittlung des Wissens: Kommunikative Gattungen. In F. Neidhardt, M. R. Lepsius, \& J. Weiß (Eds.), Kultur und Gesellschaft. Kölner Zeitschrift für Soziologie und Sozialpsychologie, 27, 190-211.

Marcoccia, M. (2004). On-line polylogues: conversation structure and participation framework in internet newsgroups. Journal of Pragmatics, 36, 115-145.

Strecker, B. (1987). Strategien des kommunikativen Handelns. Zur Grundlegung einer Grammatik der Kommunikation (Sprache der Gegenwart). Düsseldorf: Schwann.

\section{Primary Sources retrieved January 10, 2020}

AI 01 https://egon-w-kreutzer.de/wp-content/uploads/2020/12/Aigner-Austritt-1.pdf

AW $01 \mathrm{https}: / / w w w . a b g e o r d n e t e n w a t c h . d e / b l o g /$ informationsfreiheit/das-interne-strategiepapier-desinnenministeriums-zur-corona-pandemie

BI 01 https://www.bmi.bund.de/SharedDocs/downloads/DE/veroeffentlichungen/2020/corona/szenarienpapier-covid19.html

BR 01 https://www.bundesregierung.de/breg-de/themen/coronavirus/merkel-zu-corona-1729780

KF 01 https://kenfm.de/der-corona-faschismus-kippt-von-anselm-lenz/

ND 01 https://neue-debatte.com/2020/12/30/walter-van-rossum-vom-tod-der-aufklarung-unter-laborbedingungen/

ND 02 https://neue-debatte.com/2020/12/25/wissenschaft-und-aufklarung-in-der-corona-krise/

RD $01 \mathrm{https}: / /$ www.youtube.com/watch?v=v-0WeHqeL5I

RT 01 https://www.rtl.de/cms/corona-lockerungen-es-geht-ein-riss-durch-unser-land-4533448.html

SM 01 http://submnml.com/Akte-RKI-Lagepapier.pdf

SP 01 https://www.spiegel.de/politik/deutschland/angela-merkel-zu-verschwoerungsideologien-angriff-auf-unsere-ganze-lebensweise-a-95cb7814-515f-48e1-8092-9384ecd22e7c

TP 01 https://www.heise.de/forum/Telepolis/Kommentare/Keine-Uebersterblichkeit-trotz-Covid/forum-464597/

TP 02 https://www.heise.de/tp/features/Von-fragwuerdigen-und-fehlinterpretierten-CoronaZahlen-4996600.html

TP 03 https://www.heise.de/forum/heise-online/Allgemein/Trollwiese/Re-Bitte-alle-im-Forum-Patientenverfuegung-unterschreiben/posting-38095145/show/

TP 04 https://www.heise.de/forum/Telepolis/Kommentare/Warum-eine-allgemeine-Impfpflichtsein-muss/forum-463797/

TS 01 https://meta.tagesschau.de/id/147079/corona-krisenkommunikation-die-politik-lernt-aufdie-harte-tour

TW 01 https://twitter.com/eskensaskia/status/1289518034621612032

TW 02 https://twitter.com/reitschuster/status/1330594704627535872 